\title{
Phase separation and charge ordering in doped manganite perovskites: Projection perturbation and mean-field approaches
}

\author{
Shun-Qing Shen and Z. D. Wang \\ Department of Physics, The University of Hong Kong, Pokfulam, Hong Kong, China
}

(Received 10 June 1999; revised manuscript received 7 October 1999)

\begin{abstract}
A theory is developed to explain various types of electronic collective behaviors in doped manganites $R_{1-x} X_{x} \mathrm{MnO}_{3}(R=\mathrm{La}, \mathrm{Pr}, \mathrm{Nd}$, etc. and $X=\mathrm{Ca}, \mathrm{Sr}, \mathrm{Ba}$, etc.). Starting from a realistic electronic model, we derive an effective Hamiltonian by utilizing the projection perturbation techniques and develop a spin-chargeorbital coherent-state theory, in which the Jahn-Teller effect and the orbital degeneracy of $e_{g}$ electrons in Mn ions are taken into account. Physically, the experimentally observed charge-ordering state and electronic phase separation are two macroscopic quantum phenomena with opposite physical mechanisms, and their physical origins are elucidated in this theory. The interplay of the Jahn-Teller effect, the lattice distortion, as well as the double-exchange mechanism leads to different magnetic structures and to different charge-ordering patterns and phase separation.
\end{abstract}

\section{INTRODUCTION}

Charge-ordering (CO) states $^{1-7}$ and electronic phase separation $^{8,9}$ (PS) are two of the macroscopic quantum phenomena observed experimentally in doped manganites $R_{1-x} X_{x} \mathrm{MnO}_{3}(R=\mathrm{La}, \mathrm{Pr}, \mathrm{Nd}$, etc. and $X=\mathrm{Ca}, \mathrm{Sr}, \mathrm{Ba}$, etc. $)$. This seems puzzling since these two phenomena have completely opposite physical mechanisms. CO near $x=0.5$ is a regular alignment of $\mathrm{Mn}^{3+}$ and $\mathrm{Mn}^{4+}$ in the real space. It is well known that the Wigner lattice is expected to be stabilized when the repulsive potential between charge carriers dominates over the kinetic energy of the carriers. In this respect $\mathrm{CO}$ is expected to form in manganites due to a strong repulsion between charge carriers. Oppositely, PS in doped manganites near $x=0$ is characteristic of two regions of richand poor-density of charge carriers with ferromagnetic (FM) and antiferromagnetic (AF) correlations, respectively. A uniform-density state is unstable when the charge carriers are subjected to a strong attractive interaction, as discussed in high- $T_{c}$ superconductors. ${ }^{10}$ It should be a strong attraction which drives the charge carriers to the electronic PS. On the other hand, various types of magnetic structures and orbital ordering states were also observed experimentally. $\mathrm{CO}$ and PS are definitely associated with these structures. For instance, $\mathrm{CO}$ with the $(\pi, \pi, 0)$ pattern occurs under the $\mathrm{C}$-type antiferromagnetic (AF) background, and PS near $x=0$ occurs under the A-type AF background. The field-induced melting effect of $\mathrm{CO}$ shows that the $\mathrm{CO}$ decreases and eventually disappears while an external magnetic field increases. ${ }^{11}$ The field destroys the AF correlation, and the disappearance of $\mathrm{CO}$ and $\mathrm{AF}$ indicates their close relations and the possible relation between AF correlation and the repulsive interaction of charge carriers. Hence experimental observations of CO and PS in manganites strongly suggest that the sign of effective interaction between the charge carriers should depend on the dopant concentrations and the magnetic structures.

There has been considerable theoretical work motivated by the experimental research on manganese oxides. Most theoretical efforts focus on understanding metallic ferromagnetism and its connection to unusual transport properties. ${ }^{12-17}$ The scenario of double-exchange mechanism is extensively accepted to explain the metallic ferromagnetism. However, we still lack a comprehensive picture for the physical origins of the PS and CO in doped manganites. In a simplified one-band model, PS was studied analytically and numerically. ${ }^{18-20}$ An attraction between the charge carriers caused by the superexchange coupling is responsible for the instability of a uniform-density state. ${ }^{19}$ In the vicinity of $x=0.5$, the mechanisms of both long-range Coulomb interaction and the particle-hole interaction for the $\mathrm{CO}$ were proposed. ${ }^{11,21,22}$ However, PS and CO cannot be explained simultaneously in the same one-band model as strong longrange Coulomb interaction does not favor forming the PS near $x=0$. Other properties, such as the layered antiferromagnetism at $x=0$ and anomaly optical conductivity, also suggest that the double degeneracy of the $e_{g}$ orbital, which is neglected in the one-band model, should be included. The phase diagrams of doped manganites, especially for magnetic and orbital ordering, have been investigated. ${ }^{23-29} \mathrm{Re}-$ cent overviews for doped manganites are seen in Refs. 30,31.

In this paper, we explore the origins of PS and $\mathrm{CO}$ in doped manganites and establish a unified theory for these two phenomena. The paper is organized as follows. An effective Hamiltonian is derived in Sec. II. Starting from a realistic electronic Hamiltonian with strong electron correlations, several virtual processes of superexchange are considered and an effective Hamiltonian is derived by means of the projective perturbation approach and Schwinger boson formalism. A theory of the spin-charge-orbital coherent state is presented in Sec. III. Close connections of the PS and the CO with various types of AF are elucidated in Sec. IV. We also show that the Jahn-Teller (JT) effect and lattice distortion play important roles in stabilizing the magnetic structures. Some discussions and a brief summary are given in Sec. V. A detailed derivation of the effective Hamiltonian up to the second order is presented in the Appendix.

\section{EFFECTIVE HAMILTONIAN: A PROJECTION PERTURBATION APPROACH}

\section{A. Model Hamiltonian}

Doped manganese oxides, $R_{1-x} X_{x} \mathrm{MnO}_{3}$, can be regarded as a mixture of $\mathrm{Mn}^{3+}\left(3 d^{4}\right)$ and $\mathrm{Mn}^{4+}\left(3 d^{3}\right)$ ions. The three 
electrons in the outer shell of $\mathrm{Mn}^{4+}$ are almost localized in the $t_{2 g}$ orbit to form a spin maximal state with $S=3 / 2$. In the manganese ion $\mathrm{Mn}^{3+}$, apart from the three localized electrons in the $t_{2 g}$ orbit, the fourth $d$ electron locates at the $e_{g}$ orbit which is doubly degenerated. $e_{g}$ electrons can become delocalized with increasing $x$. From the above reasoning, an electronic Hamiltonian with orbital degeneracy is put forward to describe the dominant electron-electron interaction of the system ${ }^{23-29}$

$$
\begin{aligned}
H_{e}= & \sum_{i j, \gamma, \gamma^{\prime}, \sigma} t_{i j}^{\gamma \gamma^{\prime}} c_{i, \gamma, \sigma^{\dagger}}^{\dagger} c_{j, \gamma^{\prime}, \sigma}-\sum_{i, \gamma} J_{H} \mathbf{S}_{i} \cdot \mathbf{S}_{i, \gamma} \\
& +\sum_{i, \gamma, \gamma^{\prime}, \sigma, \sigma^{\prime}}\left(1-\delta_{\gamma, \gamma^{\prime}} \delta_{\sigma, \sigma^{\prime}}\right) U_{\gamma \gamma^{\prime}} \mathbf{N}_{i, \gamma, \sigma} \mathbf{N}_{i, \gamma^{\prime}, \sigma^{\prime}} \\
& -\sum_{i, \gamma \neq \gamma^{\prime}, \sigma, \sigma^{\prime}} J\left(c_{i, \gamma, \sigma^{\prime}}^{\dagger} c_{i, \gamma, \sigma^{\prime}} c_{i, \gamma^{\prime}, \sigma^{\prime}}^{\dagger} c_{j, \gamma^{\prime}, \sigma}\right. \\
& +c_{\left.i, \gamma, \sigma^{\dagger} c_{i, \gamma^{\prime}, \sigma^{\prime}}^{\dagger} c_{i, \gamma, \sigma^{\prime}}^{\dagger} c_{i, \gamma^{\prime}, \sigma^{\prime}}\right)}
\end{aligned}
$$

where $c_{i, \gamma, \sigma}^{\dagger}$ and $c_{j, \gamma, \sigma}$ are the creation and annihilation operators of $e_{g}$ electrons at the orbit $\gamma(=z$ or $\bar{z}$ where and $|z\rangle \propto\left(3 z^{2}-r^{2}\right) / \sqrt{3}$ and $|\bar{z}\rangle \propto x^{2}-y^{2}$, respectively) of site $i$ with spin $\sigma(=\uparrow, \downarrow)$, respectively. $\mathbf{N}_{i, \gamma, \sigma}=c_{i, \gamma, \sigma}^{\dagger} c_{i, \gamma, \sigma} . \mathbf{S}_{i, \gamma}$ is the spin operator of the $e_{g}$ electron and $\mathbf{S}_{i}$ is the total maximal spin operator of the three $t_{2 g}$ electrons. Here the transfer integrals in the model are assumed to take a SlaterKoster form given by the hybridization between the $e_{g}$ orbit and nearest oxygen $p$ orbit, and the model has been extensively studied to understand physics of doped manganite provskites.

Besides the Hamiltonian of the conduction electrons, we need take into account other parts of the interaction which are believed to affect the phase diagram of the doped manganites. First, a tiny hopping between $t_{2 g}$ electrons produces a weak superexchange AF coupling $H_{A F}$ :

$$
H_{A F}=J_{A F} \sum_{i j}\left(\mathbf{S}_{i} \cdot \mathbf{S}_{j}-S^{2}\right) .
$$

Apart from the electronic part of interactions, the JT effect leads to a distortion and mixes $e_{g}$ orbits. ${ }^{34}$ According to Kanamori, ${ }^{35}$ at $x=0$, the primary lattice distortion is a staggered $(\pi, \pi, \pi)$ tetragonal distortion of the oxygen octahedra surrounding the Mn sites, driven by a Jahn-Teller splitting of the outer Mn $d$ levels. Kanamori's deduction was subsequently confirmed by more detailed studies of the structure. ${ }^{36}$ Hence an effective Hamiltonian is introduced phenomenologically for the JT effect,

$$
H_{J T}=k \sum_{i, \sigma, j, \sigma^{\prime}}\left(\mathbf{N}_{i, \alpha, \sigma}-\mathbf{N}_{i, \bar{\alpha}, \sigma}\right)\left(\mathbf{N}_{j, \alpha, \sigma^{\prime}}-\mathbf{N}_{j, \bar{\alpha}, \sigma^{\prime}}\right) .
$$

where $\alpha$ depends on the direction of $\mathbf{r}_{i}-\mathbf{r}_{j}$. The tetragonal crystal field $H_{z}$ will lead to the anisotropic magnetic structure of the system: ${ }^{37}$

$$
H_{z}=-\epsilon_{z} \sum_{i, \sigma}\left(\mathbf{N}_{i, z, \sigma}-\mathbf{N}_{i, \bar{z}, \sigma}\right) .
$$

These two parts of the interaction are independent of spin.
In short, combining the JT effect and the lattice distortion caused by the crystal field, the total effective Hamiltonian is found to be

$$
H_{\text {total }}=H_{e}+H_{A F}+H_{J T}+H_{z} .
$$

\section{B. Projection perturbation approach: the strong Hund coupling case}

The model in Eq. (1) contains several parameters. It is almost impossible to obtain a complete phase diagram in the whole model parameter space. However, it was already realized that the Hund's rule coupling $J_{H}$, between the $e_{g}$ electron and three $t_{2 g}$ electrons, is very strong, which is the main origin of metallic ferromagnetism in the range of $0.2<x$ $<0.5$. $^{12}$ Most $e_{g}$ electrons favor forming $S=2$ spins with localized $t_{2 g}$ electrons in $\mathrm{Mn}^{3+}$. Usually only the strong Hund's rule coupling $J_{H}$ is taken into account. However, the on-site Coulomb interaction $U$ (intraorbit $\gamma=\gamma^{\prime}$ ) and $U^{\prime}$ (interorbits $\gamma \neq \gamma^{\prime}$ ) are also dominant energy scales and usually larger than $J_{H} S$. We will see that the strong on-site correlation play an essential role of the electronic collective behaviors. In this paper, we shall focus on the case of strong Hund coupling.

In the large $J_{H}$ limit and when the number of electrons is not greater than the number of lattice sites, each site is occupied by at most one $e_{g}$ electron. And the electron must form a spin $S+1 / 2$ state with the localized spin on the same site. The process can be realized by introducing the projection operator

$$
P=\prod_{i}\left(P_{i h}+\sum_{\gamma= \pm} P_{i \gamma s}^{+}\right) .
$$

For any state $|\alpha\rangle, P|\alpha\rangle$ is the component of the state with holes and single occupancies with spin $S+1 / 2$. This technique has been applied extensively to study the one-band Kondo lattice model. ${ }^{12,38}$ The projection operators are defined by

$$
\begin{gathered}
P_{i, h}=\prod_{\gamma, \sigma}\left(1-n_{i, \gamma, \sigma}\right), \\
P_{i, s}=\sum_{\gamma, \sigma} n_{i, \gamma, \sigma} \prod_{\gamma^{\prime}, \sigma^{\prime} \neq \gamma, \sigma}\left(1-n_{i, \gamma^{\prime}, \sigma^{\prime}}\right), \\
P_{i, \gamma, s}^{+}=\sum_{\sigma, \sigma^{\prime}}\left(\frac{\mathbf{S}_{i} \cdot \boldsymbol{\sigma}+(S+1) \mathbf{I}}{2 S+1}\right)_{\sigma \sigma^{\prime}} c_{i, \gamma, \sigma}^{\dagger} c_{i, \gamma, \sigma^{\prime}} P_{i, s} .
\end{gathered}
$$

Hence, in the infinite $J_{H}$ limit, the Hamiltonian in Eq. (1) is reduced to

$$
H_{e}^{(a)}=P H_{e} P
$$

as shown in Fig. 1(a). This is the double-exchange model with orbital degeneracy. It is worth mentioning that the double occupancy with different orbital indices is also excluded. With the help of the Schrieffer-Wolff transformation, ${ }^{39}$ the finite, but large $J_{H}$ effect is taken into account by superexchange processes in the second-order projective perturbation approach: (1) $\mathrm{Mn}^{3+} \mathrm{Mn}^{4+}$ $\Leftrightarrow \mathrm{Mn}^{4+} \mathrm{Mn}^{3+}$ as shown in Fig. 1(b) and (2) $\mathrm{Mn}^{3+} \mathrm{Mn}^{3+}$ 

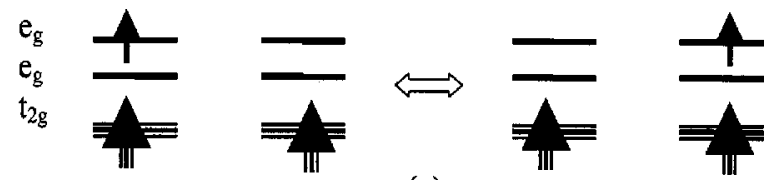

(a)
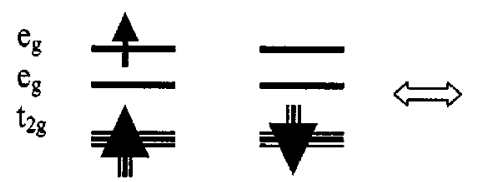

(b)
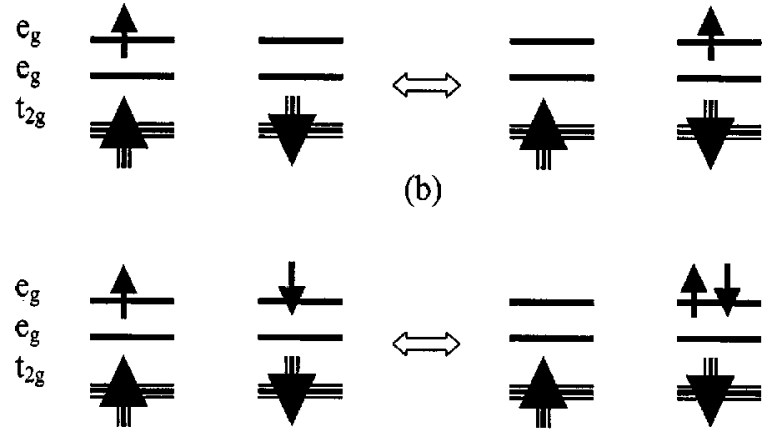

(c)
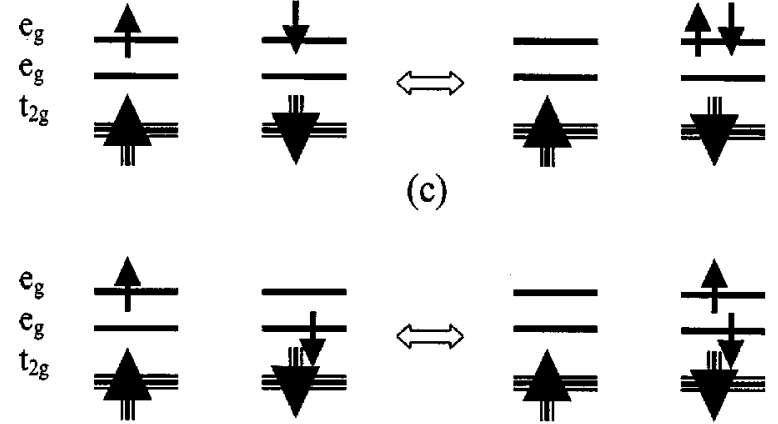

(d)
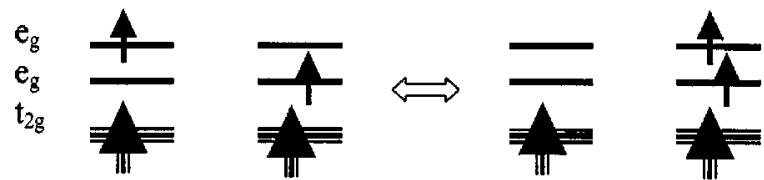

(e)
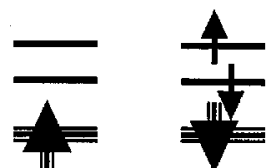

FIG. 1. Five processes that generate the effective Hamiltonian along the $z$ axis. (a) a direct hopping of $e_{g}$ electron from one site to its nearest-neighbor site. The spin of electron must be parallel to the localized spin at the same site. (b)-(e) are four virtual processes to the superexchange couplings: (b) the intermediate state is a single occupancy with spin $S-1 / 2$; (c) the intermediate state is a double occupancy on the same orbit; (d) the intermediate state is a double occupancy with spin $S+1$ on different orbits; and (e) the intermediate state is a double occupancy with spin $S$ on different orbits. These five processes correspond to the five terms in Eq. (6), respectively. The interactions along other $x$ and $y$ axes are derived by using the symmetry of rotation.

$\Leftrightarrow \mathrm{Mn}^{4+} \mathrm{Mn}^{2+}$ as shown in Figs. 1(c)-1(e). After considering the second-order perturbation correction to Eq. (4), we obtain

$$
H_{e f f}=P H P-\sum_{\alpha} \frac{1}{\Delta E_{\alpha}} P H Q_{\alpha} H P,
$$

where $\Delta E_{\alpha}$ are the energy difference of the intermediate state and the initial state as shown in Figs. 1(b)-1(e). $Q_{\alpha}$ $(\alpha=b, c, d, e)$ are the projection operators for the intermediate states. The derivation of Eq. (5) and the explicit expressions of $\Delta E_{\alpha}$ and $Q_{\alpha}$ are presented in the Appendix.

The technique to derive the effective Hamiltonian here has been used by several authors. ${ }^{25,29}$ As the strong on-site Hund coupling and Coulomb interactions, the model in Eq. (1) is one of the strongly correlated electron system. What we do here follows the logic from the one-band Hubbard model to the famous $t-J$ model for cuprates. The strong cor- relation among electrons has been taken into account. The effective Hamiltonian holds when the excitation energies and/or the temperatures are much lower than the energy gaps $\Delta E_{a}$. Usually, in the case of strong correlation, we expect that the effective Hamiltonian in Eq. (5) and the Hamiltonian in Eq. (1) describe the same low-temperature physics. Mathematically, the Hamiltonians in Eqs. (1) and (5) are mathematically identical in the strong Hund coupling limit, $J_{H} S$ $\rightarrow+\infty$, since, in the limit, all spins of electrons are forced to be aligned along the local spins on the same sites. The projection operator can realize this constraint. For a finite and large $J_{H} S$, they are expected to describe the same physics at low temperatures (i.e., $k T \ll \Delta E_{\alpha}$ ) up to the second order of correction $t / J_{H} S$ based on the spirit of perturbation theory.

\section{Effective Hamiltonian in Schwinger boson representation}

To simplify the notations, we express the Hamiltonian in the Schwinger-boson representation. ${ }^{32}$ The representation was introduced to describe the one-band double exchange by Sarker, ${ }^{33}$ and here we generalize it to the our model by introducing another type of boson for orbital degrees of freedom. Define

$$
P_{i, \gamma, s}^{+} c_{i, \gamma, \sigma}^{\dagger} P_{i, \gamma, s}^{+}=\frac{1}{\sqrt{2 S+1}} a_{i, \sigma}^{\dagger} b_{i, \gamma}^{\dagger} f_{i}^{\dagger},
$$

where $a_{i, \sigma}^{\dagger}$ and $a_{i, \sigma}$ are the Schwinger boson operators for spin

$$
\begin{gathered}
\mathbf{S}_{i}^{+}=a_{i, \uparrow}^{\dagger} a_{i, \downarrow}, \\
\mathbf{S}_{i}^{-}=a_{i, \downarrow}^{\dagger} a_{i, \uparrow}, \\
\mathbf{S}_{i}^{z}=\frac{1}{2}\left(a_{i, \uparrow}^{\dagger} a_{i, \uparrow}-a_{i, \downarrow}^{\dagger} a_{i, \downarrow}\right) .
\end{gathered}
$$

$b_{i, \alpha}^{\dagger}$ and $b_{i, \alpha}$ are the Schwinger boson operators for orbital degrees of freedom with $\alpha=x, y, z$, which depends on the direction of $\mathbf{r}_{i j} \cdot b_{i, z}^{\dagger}|0\rangle=|z\rangle$ and $b_{i, \bar{z}}^{\dagger}|0\rangle=|\bar{z}\rangle$. The other two components are not independent and are related to the $z$ component by a transformation:

$$
\begin{aligned}
& b_{i x}=\frac{1}{2} b_{i, z}-\frac{\sqrt{3}}{2} b_{i, \bar{z}}, \\
& b_{i \bar{x}}=\frac{\sqrt{3}}{2} b_{i, z}+\frac{1}{2} b_{i, \bar{z}}, \\
& b_{i y}=\frac{1}{2} b_{i, z}+\frac{\sqrt{3}}{2} b_{i, \bar{z}}, \\
& b_{i \bar{y}}=-\frac{\sqrt{3}}{2} b_{i, z}+\frac{1}{2} b_{i, \bar{z}} .
\end{aligned}
$$

$f_{i}^{\dagger}$ and $f_{i}$ are fermion operators for the charge carrier: $n_{i}$ $=f_{i}^{\dagger} f_{i}=1$ means that there is one charge $e$ on the site, i.e., 
$\mathrm{Mn}^{3+}$, and $n_{i}=0$ means $\mathrm{Mn}^{4+}$. The constraints for the Schwinger boson and fermions are

$$
\begin{gathered}
a_{i, \uparrow}^{\dagger} a_{i, \uparrow}+a_{i, \downarrow}^{\dagger} a_{i, \downarrow}=2 S+n_{i}, \\
n_{i}^{\alpha}+n_{i}^{\bar{\alpha}}=n_{i} .
\end{gathered}
$$

In the representation, the effective Hamiltonian for conduction electrons is written as

$$
H_{e}=H_{e}^{(a)}+H_{e}^{(b)}+H_{e}^{(c)}+H_{e}^{(d)}+H_{e}^{(e)} .
$$

Each term corresponds to one of the processes shown in Fig. 1 , and is expressed as

$$
\begin{gathered}
H_{e}^{(a)}=-\sum_{i j, \sigma} \frac{t}{2 S+1} a_{i, \sigma}^{\dagger} a_{j, \sigma} b_{i, \alpha}^{\dagger} b_{j, \alpha} f_{i}^{\dagger} f_{j}, \\
H_{e}^{(b)}=\frac{2 S t^{2}}{J_{H}(2 S+1)^{2}} \sum_{i j}\left(\frac{\mathbf{S}_{i} \cdot \widetilde{\mathcal{S}}_{j}-S(S+1 / 2)}{2 S(S+1 / 2)}\right) P_{i h} P_{j \alpha}, \\
H_{e}^{(c)}=\frac{t^{2}}{U+J_{H} S} \sum_{i j}\left(\frac{\widetilde{\mathcal{S}}_{i} \cdot \widetilde{\mathcal{S}}_{j}-(S+1 / 2)^{2}}{2(S+1 / 2)^{2}}\right) P_{i j}^{s}, \\
H_{e}^{(d)}=\frac{t^{2}}{U^{\prime}+\frac{3 J}{2}+J_{H} S} \sum_{i j}\left(\frac{\widetilde{\mathcal{S}}_{i} \cdot \widetilde{\mathcal{S}}_{j}-(S+1 / 2)^{2}}{2(S+1 / 2)(S+1)}\right) P_{i j}^{d}, \\
H_{e}^{(e)}=-\frac{t^{2}}{U^{\prime}-\frac{J}{2}} \sum_{i j}\left(\frac{\widetilde{\mathcal{S}}_{i} \cdot \widetilde{\mathcal{S}}_{j}+(S+1 / 2)(S+3 / 2)}{2(S+1 / 2)(S+1)}\right) P_{i j}^{d},
\end{gathered}
$$

where $\mathbf{S}_{i}$ is a spin operator with $S$, and $\widetilde{\mathcal{S}}_{i}$ is a spin operator with $S+1 / 2$ as a FM combination of the localized spin and itinerant electron at the same site. The operators $P$ are the projection operators for charge and orbits:

$$
\begin{gathered}
P_{i j}^{s}=n_{i}^{\alpha} n_{j}^{\alpha}, \\
P_{i j}^{d}=n_{i}^{\alpha} n_{j}^{\bar{\alpha}}, \\
P_{i h}=1-n_{i}, \\
P_{i \alpha}=n_{i}^{\alpha},
\end{gathered}
$$

where $n_{i}^{\alpha}=b_{i, \alpha}^{\dagger} b_{i, \alpha}$. Finally, it is worth mentioning that we just keep the two-site interactions and neglect three-site interactions in Eq. (6). The three-site terms describe indirect hopping processes between the next-nearest-neighbor site via the intermediate states and is believed to be relatively small compared with the direct hopping terms. Horsch et al. ${ }^{29}$ derived an effective Hamiltonian with a general hopping term in the ferromagnetic phase. If we take a Slater-Koster form, their Hamiltonian coincides with ours in the ferromagnetic phase.
The other terms in Eq. (2) become

$$
\begin{gathered}
H_{A F}=J_{A F} \sum_{i j}\left(\overline{\mathbf{S}}_{i} \cdot \overline{\mathbf{S}}_{j}-S^{2}\right), \\
H_{J T}=k \sum_{i j}\left(n_{i}^{\alpha}-n_{i}^{\bar{\alpha}}\right)\left(n_{j}^{\alpha}-n_{j}^{\bar{\alpha}}\right), \\
H_{z}=-\epsilon_{z} \sum_{i}\left(n_{i}^{z}-n_{i}^{\bar{z}}\right),
\end{gathered}
$$

respectively, where

$$
\overline{\mathbf{S}}_{i}=\mathbf{S}_{i}\left(1-n_{i}\right)+\frac{2 S}{2 S+1} \widetilde{\mathcal{S}}_{i} n_{i}
$$

Hence, up to the order of $t^{2} / J_{H}$ the total effective Hamiltonian in the representation of the Schwinger bosons for both spin and orbit is

$$
H_{e f f}=H_{e}+H_{A F}+H_{J T}+H_{z} .
$$

Here each term should be restricted in the projected space. Approximately, $H_{e f f}$ in Eq. (7) and $H_{\text {total }}$ in Eq. (2) are expected to describe the same low-energy physics in the large $J_{H}$ case.

The present theory is based on Eq. (7), in terms of which we are able to establish a unified description for the electronic behaviors in doped manganites for the first time. In this paper, the model parameters are roughly estimated from the excitation energies of $\mathrm{Mn}$ ions and the density-functional calculations: $^{40}$ we take $t=0.41 \mathrm{eV}$ as energy unit. $2 t / J_{H}(2 S+1)=0.35 ; \quad t /\left(U+J_{H} S\right)=0.042 ; \quad t /\left(U^{\prime}+\frac{3}{2} J\right.$ $\left.+J_{H} S\right)=0.056 ; t /\left(U^{\prime}-\frac{1}{2} J\right)=0.106$; and $J_{A F}=0.001$. All the phase diagrams in this paper are established on this set of parameters.

\section{SPIN-CHARGE-ORBITAL COHERENT-STATE FORMALISM}

The second-order projection perturbation approach includes part of the strong correlation between conduction electrons and the localized spin, and removes the direct Hund coupling $J_{H}$. Some properties of the model [Eq. (7)] become clearer than the original one [Eq. (1)]. For instance, $H_{e}^{(a)}$ describes the double-exchange mechanism for ferromagnetism; $H_{e}^{(b)}$ describes a particle-hole interaction with an AF coupling; $H_{e}^{(c)}$ and $H_{e}^{(d)}$ describe the AF superexchange couplings with the same and different orbitals, respectively, and $H_{e}^{(e)}$ describes a FM superexchange coupling with different orbitals. Each term becomes predominate at some point in the phase space. However, the effective Hamiltonian [Eq. (7)] still seems to be very complicated, and it is still very hard to fix its physical properties. From the study of the famous $t$ - $J$ model, which is derived from the one-band Hubbard model by the same projection perturbation, we learn that this is just a first step to understand the physics of the electronic Hamiltonian in Eq. (1) in the strong Hund coupling case. 


\section{A. Spin-charge-orbital coherent state}

To investigate the effective Hamiltonian, we apply the spin-coherent state mean-field theory. We introduce two polar parameters $\theta_{i}$ and $\phi_{i}$ for spin bosons at the site $i$, and two parameters $\alpha_{i}$ and $\beta_{i}$ for orbital bosons. Following Auerbach, ${ }^{41}$ we define the spin-charge-orbital coherent state

$$
\begin{gathered}
\left|\theta_{i}, \phi_{i}, \alpha_{i}, \beta_{i}, \xi_{i}\right\rangle=\left|\theta_{i}, \phi_{i}\right\rangle_{S}|0\rangle+\left|\theta_{i}, \phi_{i}\right\rangle_{S+1 / 2} \\
\otimes\left|\alpha_{i}, \beta_{i}\right\rangle_{1 / 2} \xi_{i} f_{i}^{\dagger}|0\rangle,
\end{gathered}
$$

where

$$
\begin{aligned}
\left|\theta_{i}, \phi_{i}\right\rangle_{S}= & \frac{1}{\sqrt{(2 S) !}} \\
& \times\left[\cos \frac{\theta_{i}}{2} e^{i \phi_{i} / 2} a_{i, \uparrow}^{\dagger}+\sin \frac{\theta_{i}}{2} e^{-i \phi_{i} / 2} a_{i, \downarrow}^{\dagger}\right]^{2 S}|0\rangle, \\
\left|\alpha_{i}, \beta_{i}\right\rangle_{1 / 2} & =\left(\cos \frac{\alpha_{i}}{2} e^{i \beta_{i} / 2} b_{i, z}^{\dagger}+\sin \frac{\alpha_{i}}{2} e^{-i \beta_{i} / 2} b_{i, z}^{\dagger}\right)|0\rangle
\end{aligned}
$$

and $\xi$ is an anticommuting Grassmann variable. Define

$$
|\Phi\rangle=\prod \otimes\left|\theta_{i}, \phi_{i}, \alpha_{i}, \beta_{i}, \xi_{i}\right\rangle .
$$

The Hamiltonian function is

$$
\mathcal{H}=\frac{\left\langle\Phi\left|H_{e f f}\right| \Phi\right\rangle}{\langle\Phi \mid \Phi\rangle}
$$

where

$$
\begin{gathered}
\mathcal{H}_{e}^{(a)}=-\sum_{i j} t\left(t_{i j}^{s} t_{i j}^{\alpha} \xi_{i}^{*} \xi_{j}+\text { H.c. }\right), \\
\mathcal{H}_{e}^{(b)}=-\frac{2 S t^{2}}{J_{H}(2 S+1)^{2}} \sum_{i j} \sin ^{2} \frac{\Theta_{i j}}{2} n_{j, \alpha}\left(1-\xi_{i}^{*} \xi_{i}\right) \xi_{j}^{*} \xi_{j}, \\
\mathcal{H}_{e}^{(c)}=-\frac{t^{2}}{U+J_{H} S} \sum_{i j} \sin ^{2} \frac{\Theta_{i j}}{2} n_{i, \alpha} n_{j, \alpha} \xi_{i}^{*} \xi_{i} \xi_{j}^{*} \xi_{j}, \\
\mathcal{H}_{e}^{(d)}=-\frac{t^{2}}{3 J} \frac{(S+1 / 2)}{2}+J_{H^{\prime} S} \\
\times \sum_{i j} \sin ^{2} \frac{\Theta_{i j}}{2} n_{i, \alpha} n_{j, \alpha} \xi_{i}^{*} \xi_{i} \xi_{j}^{*} \xi_{j}, \\
\mathcal{H}_{e}^{(e)=-} \frac{t^{2}}{U^{\prime}-\frac{J}{2}} \sum_{i j}\left(\frac{(S+1 / 2) \cos \Theta_{i j}+(S+3 / 2)}{2(S+1)}\right. \\
\times n_{i, \alpha} n_{j, \alpha} \xi_{i}^{*} \xi_{i} \xi_{j}^{*} \xi_{j}, \\
\mathcal{H}_{A F}=-2 J_{A F} S^{2} \sum_{i j} \sin ^{2} \frac{\Theta_{i j}}{2},
\end{gathered}
$$

$$
\begin{gathered}
\mathcal{H}_{J T}=k \sum_{i j}\left(n_{i, \alpha}-n_{i, \bar{\alpha}}\right)\left(n_{j, \alpha}-n_{j, \bar{\alpha}}\right) \xi_{i}^{*} \xi_{i} \xi_{j}^{*} \xi_{j}, \\
\left.\mathcal{H}_{z}=-\epsilon_{z} \sum_{i}\left(n_{i, z}-n_{i, \bar{z}}\right) \xi_{i}^{*} \xi_{i}\right),
\end{gathered}
$$

where

$$
t_{i j}^{s}=\cos \frac{\theta_{i}}{2} \cos \frac{\theta_{j}}{2} e^{i \frac{\phi_{i}-\phi_{j}}{2}}+\sin \frac{\theta_{i}}{2} \sin \frac{\theta_{j}}{2} e^{-i\left(\phi_{i}-\phi_{j}\right) / 2},
$$$$
t_{i j}^{x}=\left(\frac{1}{2} \cos \frac{\alpha_{j}}{2} e^{-i \beta_{j} / 2}-\frac{\sqrt{3}}{2} \sin \frac{\alpha_{j}}{2} e^{i \beta_{j} / 2}\right)
$$$$
\times\left(\frac{1}{2} \cos \frac{\alpha_{i}}{2} e^{i \beta_{i} / 2}-\frac{\sqrt{3}}{2} \sin \frac{\alpha_{i}}{2} e^{-i \beta_{i} / 2}\right),
$$$$
t_{i j}^{y}=\left(\frac{1}{2} \cos \frac{\alpha_{j}}{2} e^{-i \beta_{j} / 2}+\frac{\sqrt{3}}{2} \sin \frac{\alpha_{j}}{2} e^{i \beta_{j} / 2}\right)
$$$$
\times\left(\frac{1}{2} \cos \frac{\alpha_{i}}{2} e^{i \beta_{i} / 2}+\frac{\sqrt{3}}{2} \sin \frac{\alpha_{i}}{2} e^{-i \beta_{i} / 2}\right),
$$$$
t_{i j}^{z}=\cos \frac{\alpha_{i}}{2} \cos \frac{\alpha_{j}}{2} e^{i\left(\beta_{i}-\beta_{j}\right) / 2},
$$

$$
\begin{gathered}
n_{i, \alpha}=\cos ^{2}\left(\frac{\alpha_{i}}{2}+\delta_{\alpha}\right)+\sin \delta_{\alpha} \sin \alpha_{i} \sin ^{2} \frac{\beta_{i}}{2}, \\
n_{i, \bar{\alpha}}=\sin ^{2}\left(\frac{\alpha_{i}}{2}+\delta_{\alpha}\right)-\sin \delta_{\alpha} \sin \alpha_{i} \sin ^{2} \frac{\beta_{i}}{2}
\end{gathered}
$$

with

$$
\cos \Theta_{i j}=\cos \theta_{i} \cos \theta_{j}+\sin \theta_{i} \sin \theta_{j} \cos \left(\phi_{i}-\phi_{j}\right)
$$

and $\delta_{x}=\pi / 3, \delta_{y}=-\pi / 3$, and $\delta_{z}=0$.

\section{B. Mean-field approximation}

$\mathcal{H}$ includes the fourth powers of Grassmann variables. These terms are hard to integrate, and an approximation is needed,

$$
f_{i}^{\dagger} f_{i} f_{j}^{\dagger} f_{j} \approx\left\langle f_{i}^{\dagger} f_{i}\right\rangle f_{j}^{\dagger} f_{j}+f_{i}^{\dagger} f_{i}\left\langle f_{j}^{\dagger} f_{j}\right\rangle-\left\langle f_{i}^{\dagger} f_{i}\right\rangle\left\langle f_{j}^{\dagger} f_{j}\right\rangle .
$$

$\left\langle f_{i}^{\dagger} f_{i}\right\rangle$ is taken to be the density of electrons in a densityuniform state, and of sublattice dependence when we consider a charge-ordering state. The polar parameters are also treated in the mean-field theory, following de Gennes. ${ }^{43}$ In his approach, the angles $Q_{\alpha}$ are used as the order parameters for the magnetic structures. The angles between two nearestneighbor spins are taken to be $(-1)^{i\left(\mathbf{r}_{i}-\mathbf{r}_{j}\right) \cdot \pi} Q_{\alpha}$. If all $Q_{\alpha}$ are equal to zero, it is a FM state. For a A-type AF, $Q_{\alpha}$ are zero among the $x-y$ plane, and $Q_{z}$ is nonzero along the $z$ axis. It is worth stressing that in this paper the antiferromagnetism along a specific direction does not mean that $Q_{\alpha}$ must be $\pi$. For a C-type AF, $Q_{z}$ is taken to be zero and $Q_{x, y}$ are nonzero. G-type AF means that all $Q_{\alpha}$ are equal, but nonzero. The parameters for orbital degrees of freedom are treated similarly as those for spins. The lattice is decomposed into $\mathrm{A}$ and $\mathrm{B}$ sublattices. On the same sublattice $(\alpha, \beta)$ are taken to 
be the same values. In this paper, we just compare several ordering structures: A-, C-, G-, and F-type structures. In this paper, we limit our discussion only in the case of $T=0$, i.e., the ground state. The phase diagrams are established by minimizing the free energy.

\section{CHARGE ORDERING AND MAGNETIC STRUCTURES}

Using the spin-charge-orbital coherent state theory, we focus on three phenomena which occur at different densities of doping: (a) ferromagnetism and A-type antiferromagnetism at $x=0$; (b) phase separation at small doping; and (c) charge ordering at $x=0.5$.

\section{A. A-type antiferromagnetism at $x=0$}

The magnetic structure of the parent compound $(x=0)$ has also very decisive impact on the electronic properties near the point, and is sensitive to the model parameters. The effective spin superexchange coupling is approximately

$$
\begin{aligned}
J_{e f f} / t= & \frac{t^{2}}{U+J_{H} S} \frac{\left\langle P_{i j}^{s}\right\rangle}{(S+1 / 2)^{2}}+\frac{S^{2}}{(S+1 / 2)^{2}} J_{A F} \\
& -\left(\frac{t^{2}}{U^{\prime}-\frac{J}{2}}-\frac{t^{2}}{U^{\prime}+\frac{3 J}{2}+J_{H} S}\right) \frac{\left\langle P_{i j}^{d}\right\rangle}{2(S+1 / 2)(S+1)},
\end{aligned}
$$

which depends not only on the model parameters, but also the orbital orderings. It is worth noticing that the factor before $\left\langle P_{i j}^{d}\right\rangle$ is always negative, and the factor before $\left\langle P_{i j}^{s}\right\rangle$ is positive. When $U$ is taken to be infinite, the factor before $\left\langle P_{i j}^{s}\right\rangle$ vanishes. In other words, the AF coupling in $H_{e}^{(c)}$ is suppressed completely. As the FM coupling in $H_{e}^{(e)}$ is always stronger than the AF coupling in $H_{e}^{(d)}$ (i.e., the factor before $\left\langle P_{i j}^{d}\right\rangle$ is always negative), the FM coupling becomes predominant, and the ground state becomes FM at low temperatures if we do not take into account the tiny AF coupling of $J_{A F} \cdot{ }^{42}$ In that case, the magnetic structure is independent of the JT effect and the lattice distortion, i.e., the orbital distribution of conduction electrons. When $U$ is finite, the strength $k$ of the JT effect and the lattice distortion coefficient $\epsilon_{z}$ affects the magnetic structure by adjusting orbital ordering: the JT effect favors forming a "Néel-type AF" orbitalorbital correlation, which enhances the FM coupling through the process (e) in Fig. 1(e), while the lattice distortion tends to force the orbital to polarize, which increases the AF coupling through processes (b) $-(d)$ in Figs. 1(b)-1(d). The phase diagram in Fig. 2 shows that the FM coupling survives at finite $U$ for a small $\epsilon_{z}$ and large $k$ and evolves into the A-type AF for a large $\epsilon_{z}$ and small $k$ (We choose the model parameters in Sec. IIIE.) Hence, the ferromagnetism at $x$ $=0$ comes from the superexchange process in Fig. 1(e), not the double-exchange mechanism in Fig. 1(a) as direct hopping is prohibited due to the strong coupling at $x=0$. The anisotropy of magnetism at $x=0$ originates from the crystal field along the $z$ direction, which forces orbital degrees to form a "FM" -like state along that direction while the " $\mathrm{AF}$ ", ordering remains among the $x-y$ planes. It is worth mentioning that the ferromagnetic superexchange mechanism appears only when the orbital degeneracy of $e_{g}$ electrons is

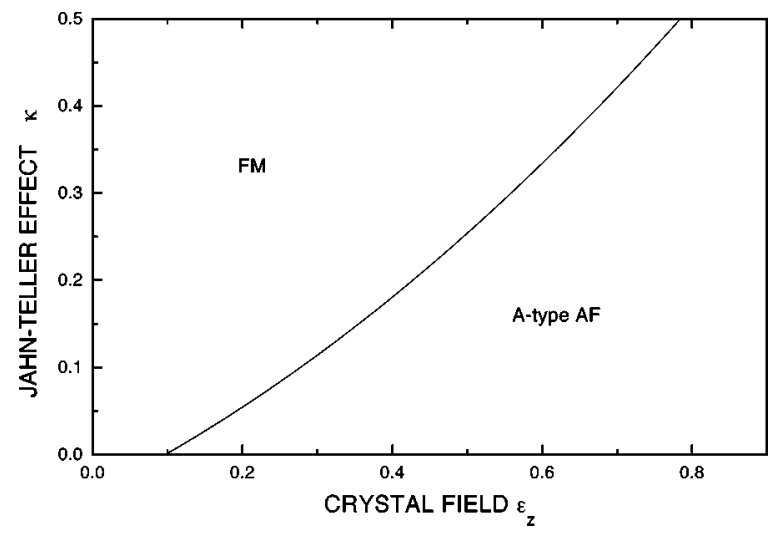

FIG. 2. The phase diagram at $x=0$.

taken into account. For a simplified one-band ferromagnetic Kondo lattice model, the ground state at half filling is always antiferromagnetic for any finite $J_{H}$ and $U .{ }^{44}$ Hence to understand the ferromagnetism, we have to take the orbital degeneracy of $e_{g}$ electrons into account such that the ferromagnetic superexchange coupling, which originates from the virtue process in Fig. 1(e), occurs, meanwhile the doubleexchange ferromagnetism is compressed completely. ${ }^{45}$

\section{B. Phase separation and A-type antiferromagnetism at $x \rightarrow 0$}

It was reported experimentally that the phase separation was observed in the single crystal of $\mathrm{La}_{1-x} \mathrm{Ca}_{x} \mathrm{MnO}_{3}$ at $x$ $=0.05$ and $0.08 .^{8}$ In the present theory, FM coupling is very strong near $x=0$ as shown Fig. 2. Under the ferromagnetic background, the AF couplings in $H_{e}$ and $H_{A F}$ are suppressed. The only interacting term which survived for polarized particles is $H_{e}^{(e)}$ in Eq. (7). Considering that the term vanishes unless the neighboring sites are occupied by two particles on different orbits at the same time, a pure attraction arises between the charge carriers. When the lattice distortion increases, the FM phase evolves into an A-type AF as the distortion forces the orbital boson to polarize along the $c$ axis, which further enhances the AF coupling via process (b). In the case of A-type AF, it is FM within the $x-y$ plane, and these FM planes are coupled antiferromagnetically. As there is no hopping between two layers with opposite spins, the system can be regarded as a reduced two-dimensional one. Phase separation was discussed in the one-band Kondo lattice model numerically and analytically ${ }^{18-20}$ and is associated with AF structure. To explore the physical origin of PS near $x=0$, the orbital degeneracy of the $e_{g}$ electrons has to be taken into account as the PS arises under the FM background. The FM near $x \rightarrow 0$ in the $x-y$ plane originates from $H_{e}^{(e)}$ in Eq. (7), i.e., the spin FM superexchange process. When the system deviates from the point slightly the magnetic structure should not change qualitatively. In fact, the PS was indeed observed in an A-type AF background. ${ }^{8}$ When the FM forms in the $x-y$ plane, the interactions for charge carriers related to AF coupling are suppressed, and the attractive interaction in the $H_{e}^{(e)}$ becomes predominant. The criteria for the phase separation is $\partial \mu / \partial n \leqslant 0$ or equivalently $\partial^{2} E / \partial x^{2} \leqslant 0$ where $E$ is the ground-state energy. On the other hand, the orbital bosons tend to form an orbital " AF"' state, which further suppresses the effective hopping 


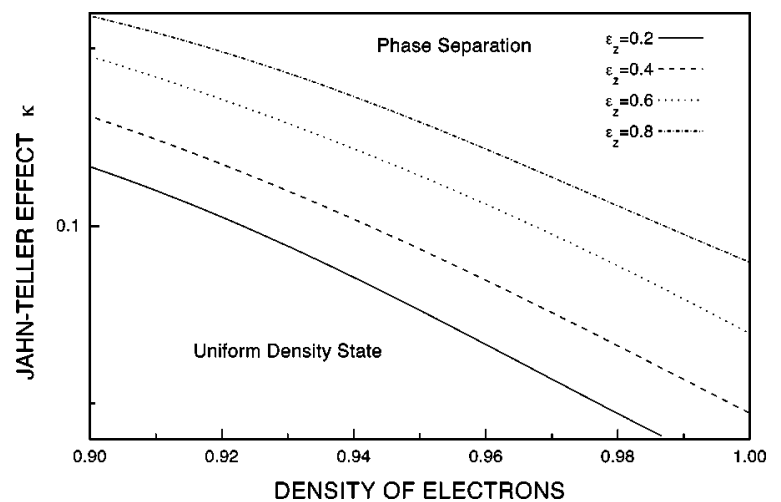

FIG. 3. Phase separation and the Jahn-Teller effect near $x=0$.

term. This property will enhance the relative ratio of the attraction to the effective hopping. The strong attraction is the physical origin of the phase separation: the charge carriers will evolve into two regimes with a high and low density of the carriers. In the case, the regime with the high density of charge carriers has a FM background, and the regime with the low density has an AF background as only the $J_{A F}$ term survives at $x=0$. Along the $c$ axis, the antiferromagnetic structure will suppress $H_{e}^{(a)}$ and $H_{e}^{(e)}$. Thus the pure interaction along the $c$ axis is repulsive. In reality, the angle between the spins on different layers is not absolutely $\pi$. So the dimensionality of A-type AF should be between 2 and 3. It is known that a higher dimensionality favors to form PS. ${ }^{46}$ The canted FM along the $c$ axis would enhance the stability of PS. The phase diagram of PS with respect to the JT effect and lattice distortion is shown in Fig. 3. When the lattice distortion increases, a stronger JT effect is required to induce PS, as expected from our general discussion. Yunoki et al. ${ }^{28}$ recently reported that the PS appears in the phase diagram of a two-dimensional model with the Monte Carlo method. Our result confirms their numerical prediction. However, the onsite Coulomb interaction was not included in their calculation.

\section{Charge ordering and antiferromagnetism at $x=0.5$}

Occurrence of the charge ordering at $x=0.5$ is associated with AF structure. For instance, the charge ordering with the $(\pi, \pi, 0)$ pattern occurs under the background of C-type AF, that is, $\mathrm{AF}$ in the $x-y$ plane and $\mathrm{FM}$ along the $c$ axis. ${ }^{1}$ The main feature of the charge ordering with $(\pi, \pi, 0)$ is that $\mathrm{Mn}^{4+}$ and $\mathrm{Mn}^{3+}$ ions aligns regularly in the $x-y$ plane and the $\mathrm{Mn}^{3+}$ ions align along the $c$ axis. It is well known that the $\mathrm{CO}$ state is expected to be stabilized when the repulsive interaction between charge carriers dominates the kinetic energy. The particle-hole interaction in the process (b) $\left[\right.$ or $H_{e}^{(b)}$ in Eq. (7)] is approximately proportional to $x(1-x)$ and reaches a minimal value at $x=0.5$. As the sign of the particle-hole interaction is negative, it is equivalent to a repulsive interaction between charge carriers (or holes). We put forward that the physical origin of the charge ordering results from this process. Of course the direct nearestneighbor Coulomb interaction is also favorable for the charge carriers to form $\mathrm{CO}$ state, as some authors discussed. ${ }^{11,21}$ The phase diagram for charge ordering at $x$ $=0.5$ shown in Fig. 4 depends on the JT effect and lattice

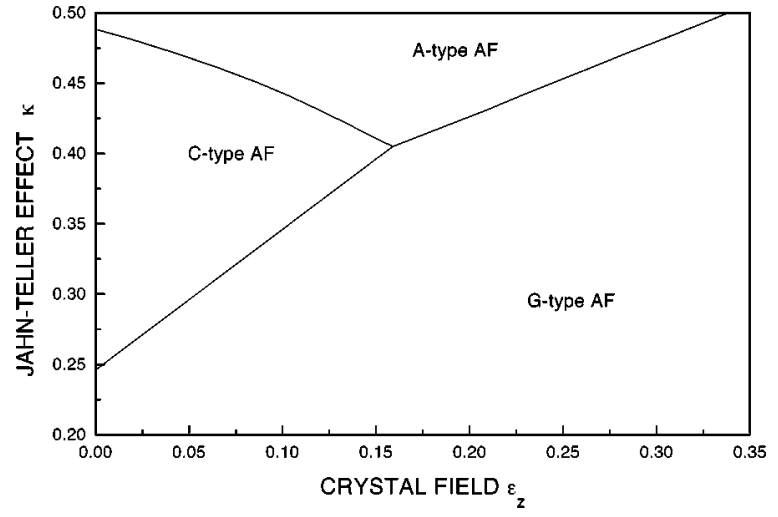

FIG. 4. The stable magnetic structure at $x=0.5$. The G-type AF has a charge carriers distribution with the pattern $(\pi, \pi, \pi)$. The C-type AF has the pattern $(\pi, \pi, 0)$. The A-type AF has a layered pattern $(0,0, \pi)$.

distortion as well as the parameters for electronic interactions. In the case of C-type AF, the particle-hole interaction in $H_{e}^{(b)}$ becomes stronger, and the hopping term is also suppressed as the $e_{g}$ electron cannot hop to a site with antiparallel spin. Relatively, the effective interaction becomes divergent when the spin-spin correlation becomes AF. In this case the state with a uniform density is unstable against the CO. To minimize the potential energy, the charge carriers tend to form the $\mathrm{CO}$ within the $x-y$ plane. Along the $c$ axis, the FM structure makes the effective interaction attractive and all charge carriers will accumulate along the axis. Therefore, the CO has the $(\pi, \pi, 0)$ pattern. When the JT effect becomes weaker and the lattice distortion increases, the $\mathrm{AF}$ coupling increases such that the FM coupling along the $c$ axis is suppressed. In this case the effective interaction along the $c$ axis also becomes repulsive. Therefore, the G-type AF with the $(\pi, \pi, \pi)$ pattern should be stable. A stronger JT effect enhances FM coupling while a stronger lattice distortion increases $\mathrm{AF}$ coupling along the $c$ axis. Thus it will force the C-type AF to evolve to the A-type AF. The charge carriers have the $(0,0, \pi)$ pattern as the interaction is repulsive for AF coupling and attractive for FM coupling.

\section{DISCUSSION AND SUMMARY}

Here we discuss the relation between the on-site $U$ and sign of effective interactions. The phase diagram in this paper is based on the parameters of the model which we list at the end of Sec. II. The parameters are roughly estimated from the excitation energies of Mn ions and the densityfunctional calculations. However, the model will contain richer phases if we adjust the model parameters. Let us first see two limits. (a) $U, U^{\prime} \gg J_{H} S, J$. From the excitation energies of the intermediate states we list in Table I, the energies in the states of Figs. 1(c) and 1(d) will be much higher than that of the state in Fig. 1(b). The FM superexchange coupling will be suppressed, and only the AF particle-hole superexchange coupling survives. In this case, it favors forming $\mathrm{CO}$ in the vicinity of $x=0.5$, but does not favor driving charge carriers to phase separate near $x=0$ as, equivalently, the net particle-particle interaction is repulsive. (b) $U \ll J_{H} S$. The intermediate state in Fig. 1(b) will have a higher energy, and the process will be suppressed. The main competition 
TABLE I. The four energy differences and projection operator $Q_{i, \alpha}$ of the intermediate states shown in Figs. 1(b)-1(e).

\begin{tabular}{ccc}
\hline \hline$\alpha$ & Energy $\Delta E_{\alpha}$ & Operator $Q_{i, \alpha}$ \\
\hline $\mathrm{b}$ & $J_{H}(2 S+1)$ & $\left(\frac{-\mathbf{S}_{i} \cdot \sigma+S \mathbf{I}}{2 S+1}\right)_{\sigma \sigma^{\prime}} c_{i, \gamma, \sigma}^{\dagger} c_{i, \gamma, \sigma^{\prime}} P_{i, s}$ \\
$\mathrm{c}$ & $\begin{array}{l}n_{i, \gamma, \uparrow} n_{i, \gamma, \downarrow} \\
\mathrm{d}\end{array} U^{\prime}+\frac{3}{2} J+J_{H} S$ & $\left(\frac{-\widetilde{\mathbf{S}}_{i} \cdot \sigma+(S+1 / 2) \mathbf{I}}{2 S+2}\right)_{\sigma \sigma^{\prime}} c_{i, \gamma, \sigma}^{\dagger} c_{i, \gamma, \sigma^{\prime}} P_{i, \bar{\gamma}, s}$ \\
$\mathrm{e}$ & $U^{\prime}-\frac{1}{2} J$ & $\left(\frac{\widetilde{\mathbf{S}}_{i} \cdot \sigma+(S+3 / 2) \mathbf{I}}{2 S+2}\right)_{\sigma \sigma^{\prime}} c_{i, \gamma, \sigma}^{\dagger} c_{i, \gamma, \sigma^{\prime}} P_{i, \bar{\gamma}, s}$
\end{tabular}

comes from processes (c) and (d). The former is AF, and the later is FM. Both of them induce an attractive interaction for charge carriers. In this case, it favors forming PS near $x$ $=0$, but does not favor forming $\mathrm{CO}$ at $x=0.5$. The density of dopants of divalent ions $X$ in $R_{1-x} X_{x} \mathrm{MnO}_{3}$ will induce structural parameters of the crystal, but should have little impact on the model-parameter-related interactions in the ions. Hence an intermediate value of the on-site interaction is very important to explain PS and CO simultaneously in the same model with the same model parameters. The parameters we used in this paper is midway between the two limits. The ratio of $U$ to $J_{H} S$ is roughly estimated to be 4.8, which indicates that the on-site interaction $U$ will also have an important impact in the formation of the rich phases in the doped manganites. Detailed discussion on the model parameters for transition-metal oxides is found in Ref. 47. It is worth mentioning that the model parameters should depend on the types and concentrations of dopants since the radii of dopant ions are different. Experimentally, some doped manganites are metallic, but some are insulating. We should be careful to estimate or choose the parameters for different specific manganites.

Next, for doped manganites, the on-site interaction is so strong that the system is one of the typical strongly correlated electron systems. In our second-order projection perturbation approach, part of the electron correlations has been taken into account, and the direct on-site Coulomb interaction and Hund's rule coupling are removed. The physical meanings in each term of the effective Hamiltonian in Eq. (7) become much clearer than that in Eq. (2). We can see clearly the physical origins of various types of magnetic structures and related physical processes. Strictly speaking, the effective Hamiltonian in Eq. (7) and the original Hamiltonian in Eq. (2) are equivalent only in the limit of large $U$ and $J_{H} S$. Up to the order of $t / U$ and $t / J_{H} S$, we expect that the two Hamiltonians describe the same physics at low temperatures based on the principle of the perturbation theory. On the other hand, although the projection technique is proved to be one of the more powerful tools to deal with strongly correlated electron systems, ${ }^{49}$ it is still a challenging problem to deal with the effective Hamiltonian. Our spinorbital-charge coherent-state theory is just an initial step for understanding the physics in doped manganites.

Finally, we come to discuss the relation of the charge inhomogeneity and the long-range Coulomb interaction. The tendency to PS and CO depends on the magnetic structures. It is anticipated that the inclusion of the longer-range Coulomb interaction will lead to a stable and microscopically inhomogeneous state. ${ }^{31}$ Recently Mori et al. reported that charge stripes arise in the range of $x>0.5$, and tend to form stripe pairs. ${ }^{48}$ It reveals strong repulsion for the charge carriers since the striped $\mathrm{Mn}^{3+}$ ions are separated by $\mathrm{Mn}^{4+}$. Our electronic model does not include the long-range Coulomb interaction. However, even if we take the interaction into account, the nearest-neighbor interaction should not be very strong such that it does not destroy the tendency to PS in the vicinity of $x=0$ caused by the superexchange attraction. Of course, it enhances the tendency to $\mathrm{CO}$ near $x$ $=0.5$ as some authors argued. ${ }^{11,21}$

In short, starting from a realistic model, we derive an effective Hamiltonian by means of the second-order projection perturbation approach in the case of the strong Hund coupling. In order to treat the model, we introduce a new type of boson for orbital degrees of freedom as well as bosons for spins and fermions for charge carriers. A spincharge-orbital coherent-state theory is presented. Physically, by adjusting the orbital ordering of the charge carriers, we find that the JT effect and the lattice distortion have a strong impact on the electronic collective behaviors as well as the magnetic structures. At the undoped case $(x=0)$, the ferromagnetism originates from the FM superexchange coupling, and the anisotropy of A-type AF is induced by the crystal field. Away from the undoped case, the FM superexchange coupling term is responsible for forming PS near the slight doping regime, while the AF particle-hole interaction drives the charge carriers to form CO states near $x=0.5$.

\section{ACKNOWLEDGMENT}

This work was supported by a CRCG/UGC research grant from the University of Hong Kong.

\section{APPENDIX: THE SECOND-ORDER PERTURBATION EXPANSION}

In this appendix, we derive the effective Hamiltonian in Eq. (5) in which the correction of the finite but large $J_{H}$ effect is taken into account. We follow Schrieffer and Wolff's method ${ }^{39}$ to derive the Kondo Hamiltonian from the periodic Anderson Hamiltonian (Also see Ref. 49). According to the projection operator $P$, the Hilbert space is divided into a subspace $P$, which consists of holes and single occupancies with spin $S+1 / 2$, and a subspace $Q(=1-P)$ with at least one double occupancy or one single occupancy with $S$ $-1 / 2$. The Schrödinger equation for the system is written as

$$
H|\phi\rangle=E_{g}|\phi\rangle,
$$

where $E_{g}$ is the ground-state energy. The equation can be expressed in the two subspaces $P$ and $Q$

$$
\begin{aligned}
& P H P|\phi\rangle+P H Q|\phi\rangle=E_{g} P|\phi\rangle, \\
& Q H P|\phi\rangle+Q H Q|\phi\rangle=E_{g} Q|\phi\rangle .
\end{aligned}
$$

The Hamiltonians $P H P$ and $Q H Q$ act within the subspaces $P$ and $Q$, respectively. $P H Q$ and $Q H P$ connect the two sub- 
spaces. To eliminate the state $Q|\phi\rangle$ in Eq. (A1), we reduce the problem in the subspace $P$ :

$$
\left(H_{P}-E_{g}\right) P|\phi\rangle=0,
$$

where

$$
H_{P}=P H P-P H Q \frac{1}{Q H Q-E_{g}} Q H P .
$$

The operator $Q$ can be expanded as

$$
\begin{aligned}
Q= & \sum_{i, \alpha} Q_{i, \alpha}\left\{\prod_{j \neq i}\left(P_{j h}+\sum_{\gamma= \pm 1} P_{j, \gamma, s}^{+}\right)\right. \\
& \left.+\sum_{j, \alpha^{\prime}} Q_{j, \alpha^{\prime}} \prod_{k \neq i, j}\left(P_{k h}+\sum_{\gamma= \pm 1} P_{k, \gamma, s}^{+}\right)+\cdots\right\} \\
= & \sum_{\alpha} Q_{\alpha},
\end{aligned}
$$

where $Q_{\alpha}$ is the projection operator in which there is at least one double occupancy or single occupancy with spin $2 S$ -1 . For our purpose, we just consider the energy correction up to the second-order perturbation. Hence we take approximately

$$
Q_{\alpha}=\sum_{i} Q_{i, \alpha} \prod_{j \neq i}\left(P_{j h}+\sum_{\gamma= \pm 1} P_{j, \gamma, s}^{+}\right) .
$$

One of the important properties is

$$
Q_{i, \alpha} Q_{i, \alpha^{\prime}}=Q_{i, \alpha} \delta_{\alpha \alpha^{\prime}} .
$$

As we are merely concerned with the low-energy excitation, the term

$$
Q_{\alpha} \frac{1}{Q H Q-E_{g}} Q_{\alpha}
$$

is replaced approximately by

$$
\frac{1}{\Delta E_{\alpha}} Q_{\alpha},
$$

where $\Delta E_{\alpha}$ is the energy difference of the energy with one $Q_{\alpha}$ and the energy of PHP. Thus the Hamiltonian is reduced to

$$
H_{e f f}=P H P-\sum_{\alpha} \frac{1}{\Delta E_{\alpha}} P H Q_{\alpha} H P .
$$

In the large- $U$ one-band Hubbard model, we have only one projection operator for the intermediate state, i.e., the operator for the double occupancy. In our case, we consider four intermediate states. The four energy differences and projection operator $Q_{i, \alpha}$ of the intermediate state shown in Figs. $1(\mathrm{~b})-1(\mathrm{e})$ are listed in the Table I.
${ }^{1}$ Z. Jirak et al., J. Magn. Magn. Mater. 53, 153 (1985).

${ }^{2}$ K. Knizek et al., J. Solid State Chem. 100, 292 (1992).

${ }^{3}$ H. Kuwahara et al., Science 270, 961 (1995).

${ }^{4}$ P. G. Radaelli et al., Phys. Rev. Lett. 75, 4488 (1995).

${ }^{5}$ A. P. Ramirez et al., Phys. Rev. Lett. 76, 3188 (1996).

${ }^{6}$ C. H. Chen and S. W. Cheong, Phys. Rev. Lett. 76, 4042 (1996).

${ }^{7}$ Y. Tokura et al., J. Appl. Phys. 79, 5288 (1996).

${ }^{8}$ G. Allodi et al., Phys. Rev. B 56, 6036 (1997).

${ }^{9}$ M. Hennion et al., Phys. Rev. Lett. 81, 1957 (1998).

${ }^{10}$ S. A. Kivelson and V. J. Emery, in Strongly Correlated Electronic Materials: Los Alamos Symposium, 1993, edited by K. S. Bedell et al. (Addison-Wesley, Reading, MA, 1994), p. 619.

${ }^{11}$ Y. Tomioka et al., Phys. Rev. Lett. 74, 5108 (1995).

${ }^{12}$ C. Zener, Phys. Rev. 82, 403 (1951).

${ }^{13}$ P. W. Anderson and H. Hasegawa, Phys. Rev. 100, 675 (1960).

${ }^{14}$ K. Kubo and N. Ohata, J. Phys. Soc. Jpn. 33, 21 (1972).

${ }^{15}$ A. J. Millis, P. B. Littlewood, and B. I. Shraiman, Phys. Rev. Lett. 74, 5144 (1995); H. Roder, J. Zhang, and A. R. Bishop, ibid. 76, 1356 (1996).

${ }^{16}$ L. Sheng, D. Y. Xing, D. N. Sheng, and C. S. Ting, Phys. Rev. Lett. 79, 1710 (1997); F. Zhong, J. Dong, and Z. D. Wang, Phys. Rev. B 58, 15310 (1998); C. M. Varma, ibid. 54, 7328 (1996).

${ }^{17}$ A. S. Alexandrov and A. M. Bratkovsky, Phys. Rev. Lett. 82, 141 (1999).

${ }^{18}$ S. Yunoki et al., Phys. Rev. Lett. 80, 845 (1998); S. Yunoki and A. Moreo, Phys. Rev. B 58, 6403 (1998).

${ }^{19}$ S. Q. Shen and Z. D. Wang, Phys. Rev. B 58, R8877 (1998).

${ }^{20}$ E. L. Nagaev, Phys. Status Solidi B 189, 9 (1994); D. P. Arovas and F. Guinea, Phys. Rev. B 58, 9150 (1998); L. J. Zhou, Q. Q.
Zheng, and H. Q. Lin, ibid. 56, 13669 (1997).

${ }^{21}$ J. D. Lee and B. I. Min, Phys. Rev. B 55, R14 713 (1997).

${ }^{22}$ S. Q. Shen and Z. D. Wang, Phys. Rev. B 59, 14484 (1999).

${ }^{23}$ K. I. Kugel and D. I. Khomskii, Zh. Éksp. Teor. Fiz. 64, 1429 (1973) [Sov. Phys. JETP 37, 725 (1973)].

${ }^{24}$ J. Inoue and S. Maekawa, Phys. Rev. Lett. 74, 3407 (1995).

${ }^{25}$ S. Ishihara, J Inoue, and S. Maekawa, Physica C 263, 130 (1997); S. Ishihara, M. Yamanaka, and N. Nagaosa, Phys. Rev. B 56, 686 (1997); R. Maezono, S. Ishihara, and N. Nagaosa, ibid. 57, R13 993 (1998).

${ }^{26}$ H. Shiba, R. Shiina, and A. Takahashi, J. Phys. Soc. Jpn. 66, 941 (1997); R. Shiina, T. Nishitani, and H. Shiba, ibid. 66, 3159 (1997).

${ }^{27}$ J. van den Brink and D. Khomskii, Phys. Rev. Lett. 82, 1016 (1999).

${ }^{28}$ S. Yunoki, A. Moreo, and E. Dagotto, Phys. Rev. Lett. 81, 5612 (1998).

${ }^{29}$ P. Horsch, J. Jaklic, and F. Mack, Phys. Rev. B 59, 6217 (1999).

${ }^{30}$ A. P. Ramirez, J. Phys.: Condens. Matter 9, 8171 (1997).

${ }^{31}$ A. Moreo, S. Yuniki, and E. Dagotto, Science 283, 2034 (1999).

${ }^{32}$ D. P. Arovas and A. Auerbach, Phys. Rev. B 38, 316 (1988); S. K. Sarker, C. Jayaprakash, H. R. Krishnamurthy, and M. Ma, ibid. 40, 5028 (1989).

${ }^{33}$ S. Sarker, J. Phys.: Condens. Matter 8, L515 (1996).

${ }^{34}$ B. Halperin and R. Englman, Phys. Rev. B 3, 1698 (1971); A. J. Millis, ibid. 53, 8434 (1996).

${ }^{35}$ K. Kanamori, J. Appl. Phys. 31, 145 (1961).

${ }^{36}$ J. B. Ellemans, B. van Laar, K. R. van der Veer, and B. O. Loopstra, J. Solid State Chem. 3, 328 (1971). 
${ }^{37} \mathrm{LaMnO}_{3}$ is an insulator which undergoes a phase transition from a cubic to tetragonal structure at $T_{s} \sim 750 \mathrm{~K}$. [See E. Wollan and W. C. Koehler, Phys. Rev. 100, 545 (1955).]

${ }^{38}$ S. Q. Shen, Phys. Lett. A 235, 403 (1997).

${ }^{39}$ J. R. Schrieffer and P. A. Wolff, Phys. Rev. 149, 491 (1966).

${ }^{40}$ J. S. Griffith, The Theory of Transition Metal Ions (Cambridge University Press, Cambridge, 1971); T. Mizokawa and A. Fujimori, Phys. Rev. B 54, 5368 (1996).

${ }^{41}$ A. Auerbach, Interacting Electrons and Quantum Magnetism (Springer-Verlag, Berlin, 1994).

${ }^{42}$ S. Q. Shen and Z. D. Wang, Phys. Rev. 59, 3291 (1999).

${ }^{43}$ P. D. de Gennes, Phys. Rev. 118, 141 (1960).

${ }^{44}$ S. Q. Shen, Phys. Rev. B 53, 14252 (1996); 55, 14330 (1997). For a review, see S. Q. Shen, Int. J. Mod. Phys. B 12, 709 (1998).
${ }^{45}$ The role of orbital degeneracy of electrons in stabilizing the ferromagnetism was realized very early. See J. C. Slater, Phys. Rev. 49, 537 (1936); J. H. van Vleck, Rev. Mod. Phys. 25, 220 (1953). A one-dimensional exact solution of ferromagnetism of an orbitally degenerated system is seen in S. Q. Shen, Phys. Rev. 57, 6474 (1998).

${ }^{46}$ V. J. Emery, S. K. Kivelson, and H. Q. Lin, Phys. Rev. Lett. 64, 475 (1990).

${ }^{47}$ M. Imada, A. Fujimori, and Y. Tokura, Rev. Mod. Phys. 70, 1039 (1998).

${ }^{48}$ S. Mori, C. H. Chen, and S. W. Cheong, Nature (London) 392, 473 (1998); A. J. Millis, ibid. 392, 438 (1998).

${ }^{49}$ P. Fulde, Electron Correlation in Molecules and Solids, 3rd Enlarged ed. (Springer-Verlag, Berlin, 1995), p. 302. 